\title{
Evaluation of Different Non-destructive Testing Methods to Detect Imperfections in Unidirectional Carbon Fiber Composite Ropes
}

\author{
Kim-Niklas Antin ${ }^{1}$ (D) Miguel A. Machado $^{2} \cdot$ Telmo G. Santos $^{2} \cdot$ Pedro Vilaça $^{1}$
}

Received: 20 August 2018 / Accepted: 6 February 2019 / Published online: 13 February 2019

(c) The Author(s) 2019

\begin{abstract}
Online monitoring of carbon fiber reinforced plastic (CFRP) ropes requires non-destructive testing (NDT) methods capable of detecting multiple damage types at high inspection speeds. Three NDT methods are evaluated on artificial and realistic imperfections in order to assess their suitability for online monitoring of CFRP ropes. To support testing, the microstructure and electrical conductivity of a carbon fiber rope is characterized. The compared methods are thermography via thermoelastic stress analysis, ultrasonic testing with commercial phased array transducers, and eddy current testing, supported by tailormade probes. While thermoelastic stress analysis and ultrasonics proved to be accurate methods for detecting damage size and the shape of defects, they were found to be unsuitable for high-speed inspection of a CFRP rope. Instead, contactless inspection using eddy currents is a promising solution for real-time online monitoring of CFRP ropes at high inspection speeds.
\end{abstract}

Keywords Non-destructive testing · Ultrasonic · Eddy current · Carbon fiber · Composite

\section{Introduction}

The use of synthetic fiber ropes has significant potential to replace steel wire ropes in civil engineering and hoisting applications. The high strength-to-weight ratio and corrosion resistance are beneficial in stationary applications such as suspended bridges [1] and offshore anchoring as well as in cyclically loaded applications like hoisting, where the rope is coiled on a drum or bent over a sheave [2]. Although the application of non-destructive testing (NDT) methods is mature and well established for metallic ropes [3], the same does not apply to synthetic fiber ropes. In addition to

Kim-Niklas Antin

kim-niklas.antin@aalto.fi

Miguel A. Machado

miguel.m@ fct.unl.pt

Telmo G. Santos

telmo.santos@fct.unl.pt

Pedro Vilaça

pedro.vilaca@aalto.fi

1 Department of Mechanical Engineering, School of Engineering, Aalto University, 02150 Espoo, Finland

2 UNIDEMI, Department of Mechanical and Industrial Engineering, NOVA School of Science and Technology, NOVA University Lisbon, 2829-516 Caparica, Portugal damage from in-service static and cyclic loading, these new kind of ropes could contain manufacturing defects and get damaged during transportation, installation or maintenance operations. Periodic visual inspection of ropes is tiresome, subjective, capable of detecting only surface defects and means downtime for the customer [3]. The purpose of this study is to find an online monitoring method that would allow condition-based maintenance, remote assessment of data and full utilization of the life of carbon fiber ropes.

Several methods for inspection of fiber ropes have been suggested, such as methods based on the electrical resistivity of the fibres [4, 5] and optical methods monitoring modal parameters [6]. X-ray inspection can give high-resolution volumetric information of the damage state of a rope [7], but inspection speeds are slow for online monitoring and there is a large volume to be inspected. Electrical resistivity works well for small samples [8], but it does not provide information on the location of damage. Furthermore, the relative increase in resistance caused by a small fraction of broken fibres is low compared to the total resistance of a long rope. It is possible to detect local changes by using embedded optical fibres [9], however, they provide only strain values, which are an indirect indication of damage. Moreover, matrix and interface damage, such as microcracking, delamination, environmental aging and debonding, does not cause an increase in strain although it can reduce 
the strength and service life of a rope [10]. Matrix cracking could be observed using electromagnetic methods if the matrix is made conductive by adding carbon nanotubes [11]. Like embedded sensors, this approach may be difficult to implement into existing products and production lines.

This paper aims to evaluate three NDT techniques, based on three different physical phenomena, with the intention of using them for continous in-service monitoring of carbon fiber ropes. The compared techniques are thermography, via thermoelastic stress analysis (TSA), ultrasonic testing and eddy current testing (ECT).

The TSA analysis is one typical method applied to polymer composites, including carbon fiber reinforced plastic (CFRP), to detect different kinds of damage, but most sensitive to delaminations [12-14]. The reflection or transmission of ultrasonic waves can be used to detect interfaces or heterogeneities in CFRP components [11]. The principle of ultrasonic inspection is similar to the thermal waves of the TSA phase image and is therefore especially sensitive to delaminations [11]. Eddy current testing [15] is typically used for inspecting undulations in carbon fiber reinforcement fabrics, quality control of stacking sequence, fiber orientation and curing effects [16]. Delamination detection has been proposed [17] and shown with artificial delaminations made with interply release film [18]. Artificial cracks made by slitting the fabric before lamination have been detected $[17,19,20]$ as well as impact damage $[16,21]$. Here, an approach is presented based on custom-made probes specifically designed with unidirectional CFRP material properties in mind. Furthermore, the special requirements of online monitoring of ropes have not been considered in the context of ECT. Namely inspection speed, contactless monitoring and sufficient distance to the monitored component. In this work, the potential of the ECT technique is enhanced by using custom-made probes.

\section{Material}

The carbon fiber rope is a structural system consisting of four pultruded unidirectional (UD) CFRP elements protected by a polyurethane coating with a thickness of about $1 \mathrm{~mm}$ (Fig. 1). Each load carrying CFRP element has a cross-section dimension of $5.0 \mathrm{~mm} \times 2.5 \mathrm{~mm}$. The CFRP elements are composite rods manufactured at an industrial production plant using the pultrusion method. The composite consists of standard modulus (high strength) polyacrylonitrile-based carbon fiber embedded in an epoxy matrix. As the elements are manufactured using a pultrusion line, they are not laminates and do not consist of plies like most CFRP structures. However, they behave in a similar manner as unidirectional (UD) laminates [6]. Like laminated structures, the microstructure of the pultruded elements contains resin rich zones, but their orientation varies. Failure due to excessive shear stresses and impact loading results in nearplanar matrix cracks that closely resemble delaminations commonly seen in laminates. Such matrix cracks are henceforth called delaminations. The delaminations are typically located in the middle of the specimen parallel to the XZplane and they extend through the width. The microstructure and damage morphology has been described in detail earlier [7].

Electrical resistivity measurements were made on the CFRP elements using different methods. The CFRP bulk resistivity was measured in three directions (XYZ) represented in Fig. 1 using a four-point probe with four tungsten carbide needles, arranged along a straight line and spaced $0.635 \mathrm{~mm}, d[\mathrm{~m}]$, from each other. A current source forced a constant electrical current $I[\mathrm{~A}]$ through the external needles. A 2182A nanovoltmeter simultaneously measured the voltage $V[\mathrm{~V}]$ produced between the inner needles. Assuming equally spaced contact points, the resistivity of a bulk material (semi-infinite in lateral dimension and with $t \gg d$ ) is given by Eq. 1 .

$\rho=\frac{2 \pi d V}{I}$

Table 1 Electrical resistivity and conductivity of the CFRP sample for XYZ directions

\begin{tabular}{llll}
\hline Direction & $\mathrm{X}$ & $\mathrm{Y}$ & $\mathrm{Z}$ \\
\hline Electrical resistivity $[\mathrm{m} \Omega \mathrm{m}]$ & 8.57 & 12.8 & 0.077 \\
Electrical conductivity $\left[\mathrm{S} \mathrm{m}^{-1}\right]$ & 117 & 78.1 & 13,000 \\
\hline
\end{tabular}

Fig. 1 Geometry of the carbon fiber rope system: a dimensions of the cross-section of the individual CFRP elements (in millimeters); $\mathbf{b} 3 \mathrm{D}$ perspective of the carbon fiber rope system with four parallel CFRP elements, protected by a polymer coating
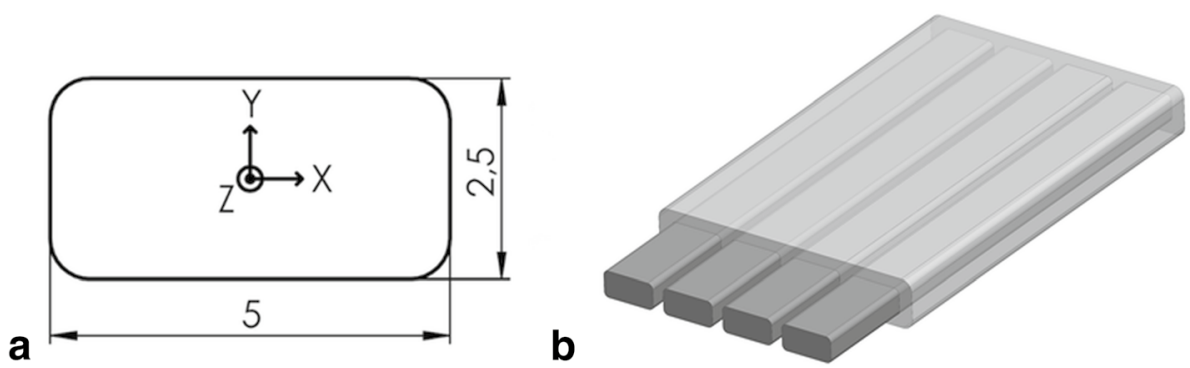

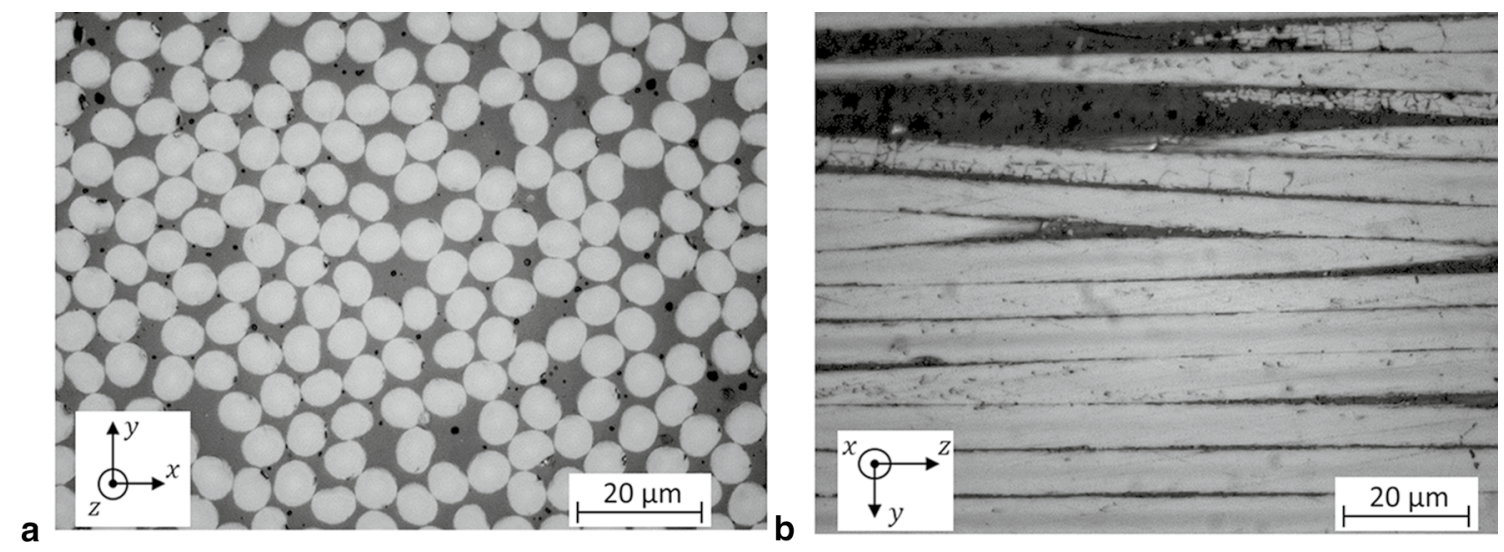

Fig. 2 Micrographs showing the contact between individual carbon fibers: a transversal view; b longitudinal view

The results obtained with the potential drop measurement are presented in Table 1 . The electrical conductivity in the longitudinal direction $(\mathrm{Z})$ is much higher than in the other two directions due to the unidirectional orientation of the fibers. However, there is also transverse bulk conductivity because the fibers are closely packed (Fig. 2). The fibers do not run in parallel either (fiber waviness), which leads to occasional contact with all its neighbours (Fig. 3).The electrical conductivity in the $\mathrm{X}$ and $\mathrm{Y}$ directions are a slightly different, because the sample length in those directions is short, which increases the measurement error.

\section{Defects}

Artificial damage was produced, and quasi-realistic damage was induced, to both the individual uncoated CFRP elements and the CFRP rope system, which comprises of four parallel CFRP elements completely surrounded by a protective polymer coating. Samples with artificial damage were produced by machining, via drilling of flat-bottomed holes in the individual uncoated CFRP elements and partial through-thickness sawing of the CFRP rope system. Internal defects can not be produced by cutting operations and therefore quasi-realistic damage was also created with mechanical loading to the individual rods, via three-point bending, inducing delamination damage, and to the whole rope system, via low-velocity drop-weight impact, inducing multiple (complex) damage types.

The flat-bottomed holes were drilled in the middle of the uncoated CFRP element with a depth of $1.0 \mathrm{~mm}$ using a high-speed steel end mill of $2.0 \mathrm{~mm}$ diameter. Rotation speed of the tool was set to $1600 \mathrm{rev} / \mathrm{min}$ and manual feed was applied in order to exert minimal force on the composite. Ethanol was periodically sprayed on the tool for lubrication and removal of carbon dust. The diameter and

a
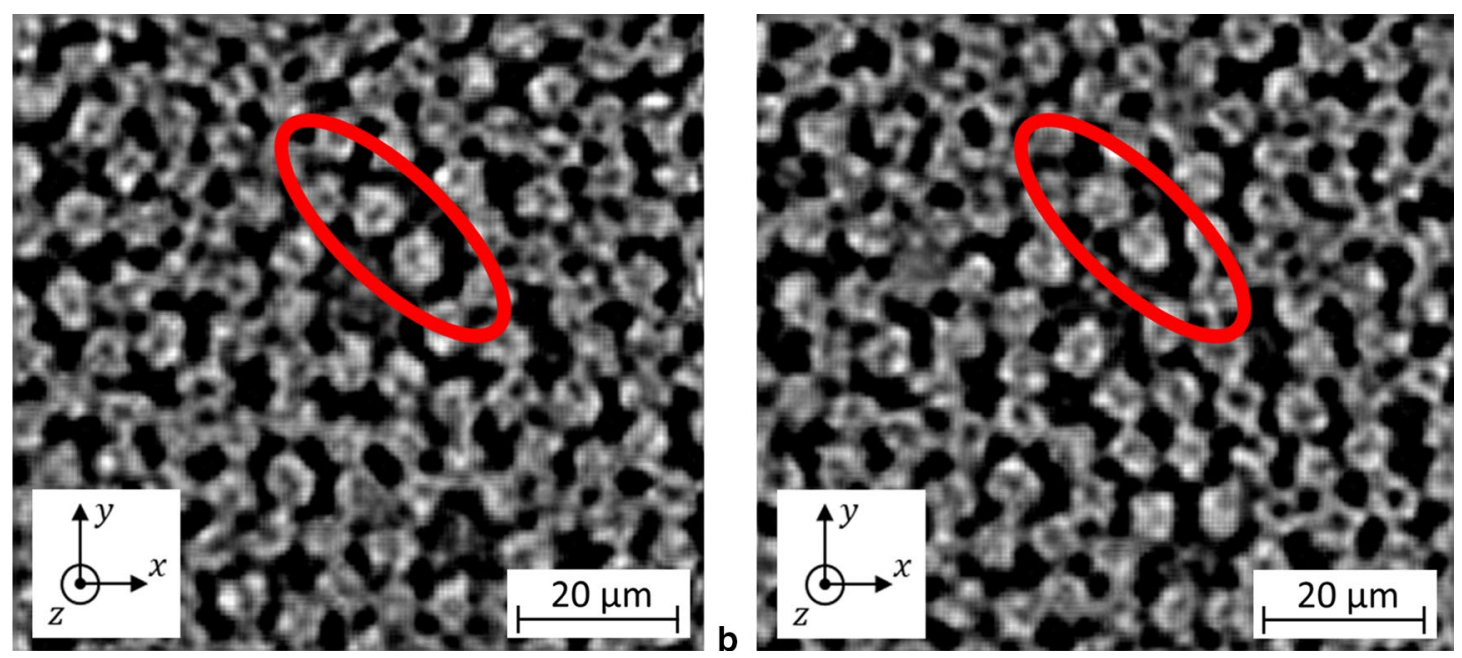

Fig. 3 X-ray microtomography $50 \mu \mathrm{m}$ apart showing the fiber waviness and contact: a transversal section $(\mathrm{Z}=0)$; b transversal section $(\mathrm{Z}=50 \mu \mathrm{m})$ 
depth of the hole was verified with optical microscopy. The size of machined defects was controllable, and the resulting defects were reproducible. However, they are not very realistic with respect to real-life applications. Therefore, three-point bending was used to induce delaminations. The loading conditions followed International Organization for Standardization (ISO) 14130 [22]. The support span was $12.8 \mathrm{~mm}$ and a roller diameter of $6.0 \mathrm{~mm}$ was used. The test speed was $1 \mathrm{~mm} / \mathrm{min}$ and the bending was interrupted when a drop in force was observed, usually accompanied by an audible crack. The delaminations typically extend through the width (X-direction) and in the case of a relatively short sample, they also propagate all the way to one end (Z-direction) of the sample (Table 2). Using longer samples, such as $1000 \mathrm{~mm}$, resulted in delaminations slightly longer than the support span.

Coated rope samples were subjected to low-velocity dropweight loading according to American Society for Testing and Materials (ASTM) D5628-10 [23]. A weight of $1530 \mathrm{~g}$ with a hemispherical $16 \mathrm{~mm}$ diameter impact head was dropped from a height corresponding to a potential energy of $15 \mathrm{~J}$. The samples were clamped to a steel frame with an $\varnothing 76 \mathrm{~mm}$ opening during the impact test, making it essentially a constrained three-point bending setup. In addition, large fiber breaks were produced by sawing a transverse cut in the sample approximately $1 \mathrm{~mm}$ deep. The width of the cut was $2 \mathrm{~mm}$.

\section{Thermography via Thermoelastic Stress Analysis (TSA)}

The TSA was implemented using a servo-hydraulic MTS test machine to load the CFRP material in cyclic uniaxial tension-tension loading. The maximum stress was $300 \mathrm{MPa}$ and a frequency of $5 \mathrm{~Hz}$ was used. Higher frequencies are recommended to ensure adiabatic conditions [12], but inertia of the hydraulic grips used may result in overshooting and inaccurate force control and therefore a lower frequency was used. The surface temperature was recorded using a FLIR X6580sc camera at 100 frames per second for $5 \mathrm{~s}$. The loading signal from the material testing machine was synchronized with the thermal waves recorded by the camera to achieve a lock-in condition. Measuring the surface temperature of the UD CFRP via TSA is challenging due to various factors, such as, anisotropy of coefficients of thermal expansion (CTE) of the composite, near-zero CTE of carbon fiber, differences in the behaviour of matrix and reinforcement, large surface temperature variations compared to the magnitude of the thermoelastic effect and variations in surface emissivity [12]. For example, extensive damage in the form of a flat-bottomed hole caused only a $0.1 \mathrm{~K}$ change in temperature (Fig. 4). However, TSA is highly sensitive to delaminations due to a local retardation of the heat flux, which leads to a phase lag of the thermal wave (Fig. 5). Lock-in condition is not necessarily needed if the local stresses are high enough and heat is generated irreversibly within the sample. Simply monitoring the average surface temperature with an inexpensive Fluke Ti32 infrared thermal imaging camera during cyclic loading $\left(\sigma_{\max }=300 \mathrm{MPa}\right.$, $f=4 \mathrm{~Hz}$ ) can reveal large damage. A $\varnothing 2 \mathrm{~mm}$ flat-bottomed hole with a depth of $1.25 \mathrm{~mm}$ causes an increase in temperature of $0.5 \mathrm{~K}$ (Fig. 6) whereas an undamaged reference sample showed no significant difference in surface temperature. Thermography did not work for specimens coated with a protective polymer coating (defects $\mathrm{C}$ and $\mathrm{D}$ in Table 2), because the coating was not transparent to the wavelengths detected by the camera. Thermography is therefore not a feasible online monitoring method for practical applications where a ductile and impact resistant coating is needed to protect the brittle CFRP material.

\section{Ultrasonic Testing}

The ultrasonic inspection was made in water immersion with an $\mathrm{X}-\mathrm{Y}$-encoder. The remaining conditions and parameters are presented in Table 3. A synchronization gate was set on the reflection from the top surface of the CFRP element to keep the measuring gate within the sample boundaries regardless of sample distance to probe. In the case of the sawed cut (defect C), the defect depth was so close to the bottom surface that the measuring gate had to include the back-wall echo as well. In that case, the absence of signal indicated damage when looking at the C-scan. A sizing curve was used to increase gain as a function of sample thickness to compensate for the attenuation especially for coated samples. The differently oriented scans were then reconstructed and exported via Olympus TomoView software.

The damage morphologies and orientation of a flat-bottomed hole in sample with defect A (Fig. 7) and delaminations in sample with defect B (Fig. 8) are especially suitable for ultrasonic inspection, due to the large interface area and therefore clear images of the defects could be generated. The signal from the delamination was stronger than the one from the flat-bottomed hole. The impact damage and sawed cut are less optimal in terms of pulse-echo type inspection executed from the top, but the $\mathrm{C}$-scans reveal damage locations and size accurately (Figs. 9, 10). The saw cut can be seen in all four parallel carbon fiber elements even when the coating causes attenuation of the signal. Although phased array ultrasonics was able to detect all damage types, inspection is relatively slow and requires contact, which makes it cumbersome for online monitoring of non-static ropes, and nearly impossible for high-speed inspection of long ropes. 
Table 2 Defects in the samples: A and B are uncoated CFRP elements; C and D are rope specimens

Defect Type of defect

A $\quad \varnothing 2 \mathrm{~mm}$ flat-bottomed hole defect produced via drilling

Delamination induced via three-point bending

C In-depth cut with penetration of $1 / 3$ thickness produced via sawing

D Multi-damage induced via low-velocity drop-weight loading (impact damage 15 J)

\section{Picture}
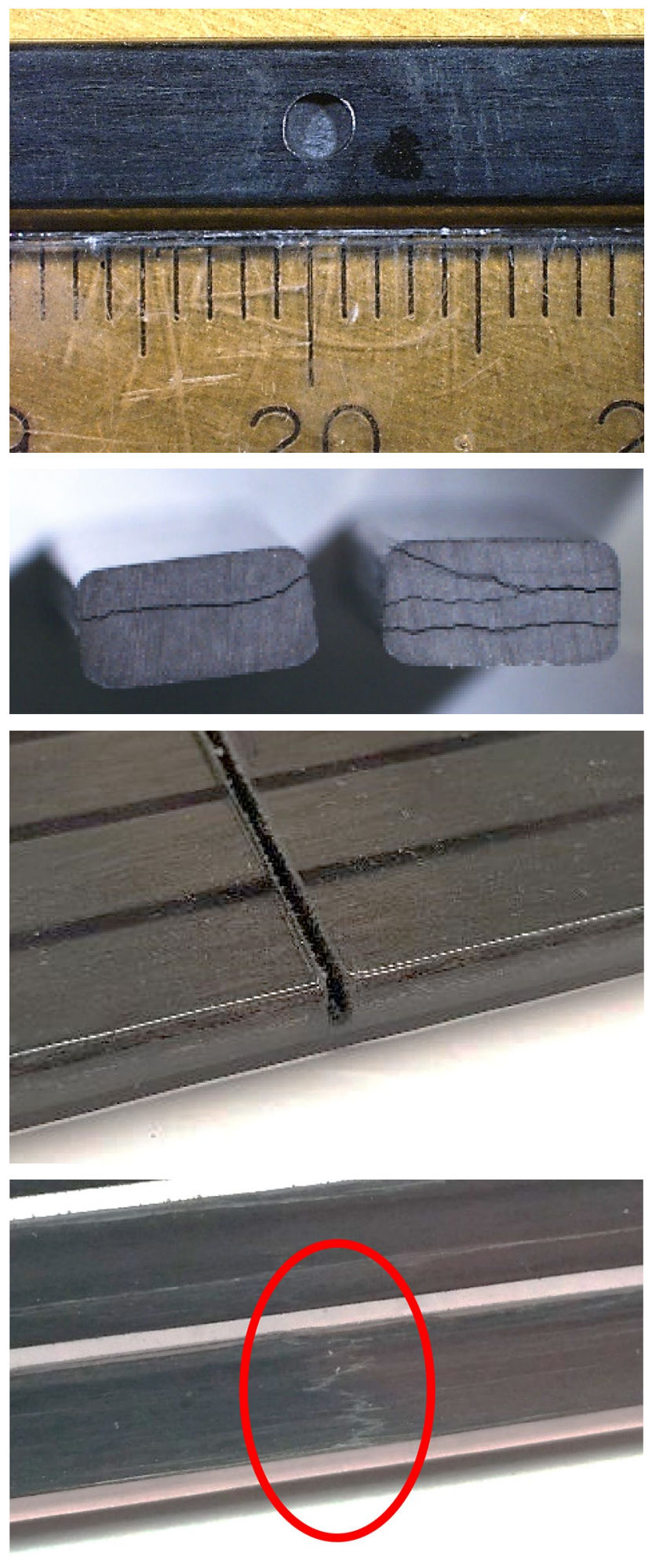


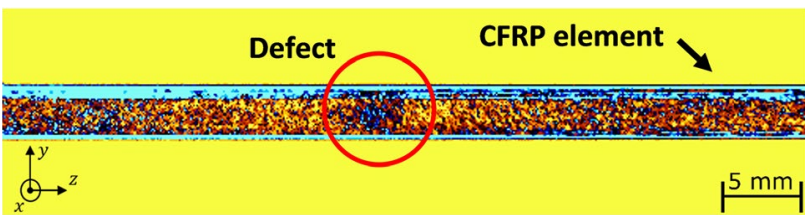

Fig. 4 TSA absolute temperature image from the top identifying the hole (defect $\mathrm{A}$ ) on the backside. Temperature range $0.1 \mathrm{~K}$

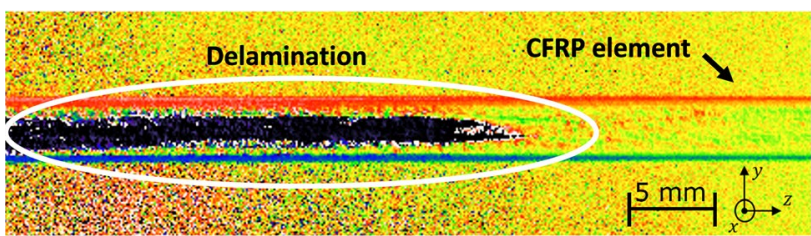

Fig. 5 TSA phase image showing a delamination (defect B) in the CFRP element. Phase range $360^{\circ}$

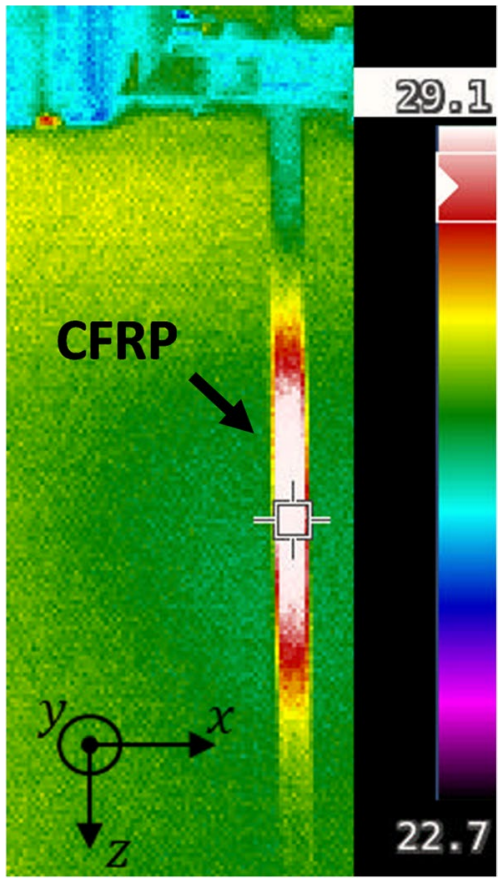

Fig. 6 Thermograph for flat-bottomed hole (defect A) on the backside, showing a local increase in average temperature $\left({ }^{\circ} \mathrm{C}\right)$ during cyclic loading

\section{Eddy Currents Testing (ECT)}

Thermography via TSA and ultrasonic testing are sensitive to interfaces interfering with wave propagation, but ECT presented here relies on using alternating current in an excitation coil, which causes a primary magnetic field, leading to
Table 3 Parameters of the ultrasonic inspection

\begin{tabular}{ll}
\hline Parameter & Value \\
\hline Detector & Olympus Omniscan MX \\
Probe & $10-\mathrm{L}-64-32 X 7-\mathrm{I} 1-\mathrm{P}-2.5-\mathrm{OM}$ \\
Operating frequency & $9.6 \mathrm{MHz}$ \\
Transmitter voltage & $40 \mathrm{~V}$ \\
Receiver gain & $18 \mathrm{~dB}$ \\
Amplitude threshold & $20 \%$ \\
Phased array & 64 elements in banks of 16 \\
& with 1 element $(0.5 \mathrm{~mm})$ \\
Line scan resolution & pitch \\
Distance to sample and focal distance & $0.5 \mathrm{~mm}$ \\
Skew angle relative to Z & $900^{\circ}$ \\
\hline
\end{tabular}

induction of eddy currents in the material under inspection. The eddy currents cause a secondary magnetic field, which is measured using a sensing coil. Damage in the conducting constituents, namely carbon fibers, can cause changes in the eddy current paths, which alters the secondary magnetic field when compared to undamaged material. However, the damage does not necessarily have to be in the conductive constituents, because the quasi-UD carbon fibers are in contact with each other, leading to transverse bulk conductivity. Therefore, delaminations could, in theory, be detected as well, although the transverse bulk conductivity is much lower than the longitudinal conductivity.

\subsection{Numerical Simulations}

Numerical simulation was performed in order to evaluate the behaviour of eddy current in the CFRP material. For this, a numerical simulation software (ANSYS Electronics) was used, and it calculates an approximate numerical solution of Maxwell's equations in their full formulation, Finite Integration Technique (FIT). The simulation comprises of one carbon fiber element and an excitation coil. The element has the same section as the individual CFRP elements, $5 \times 2.5$ $\mathrm{mm}$, and $100 \mathrm{~mm}$ length. The excitation coil was placed $3 \mathrm{~mm}$ above the element to allow some clearance even in the presence of a protective coating and $1 \mathrm{~A}$ current was used with a frequency of $6 \mathrm{MHz}$. The excitation element section used was $1 \times 1 \mathrm{~mm}$ where 10 parallel conductors pass. In Fig. 11 is depicted the model used and the tetrahedral mesh representation which contains 1.700 .000 elements with a mesh refinement in the carbon fiber. An isotropic material (aluminium) was first considered in order to understand the different behaviour of eddy current in an isotropic and anisotropic material.

Figure 12 shows the current density in one structural element when different excitation orientatione are used and 
Fig. 7 Results from the ultrasonic testing of sample with hole (defect A): C-scan and B-scan

Fig. 8 Results from the ultrasonic testing of sample with defect B (delamination): C-scan and B-scan

Fig. 9 Results from the ultrasonic testing of sample with defect $\mathrm{C}$ (coated rope system with sawed CFRP elements): $\mathrm{C}$-scan and B-scan

Fig. 10 Results from the ultrasonic testing of sample with defect $\mathrm{D}$ (coated rope with impact damage): C-scan and B-scan
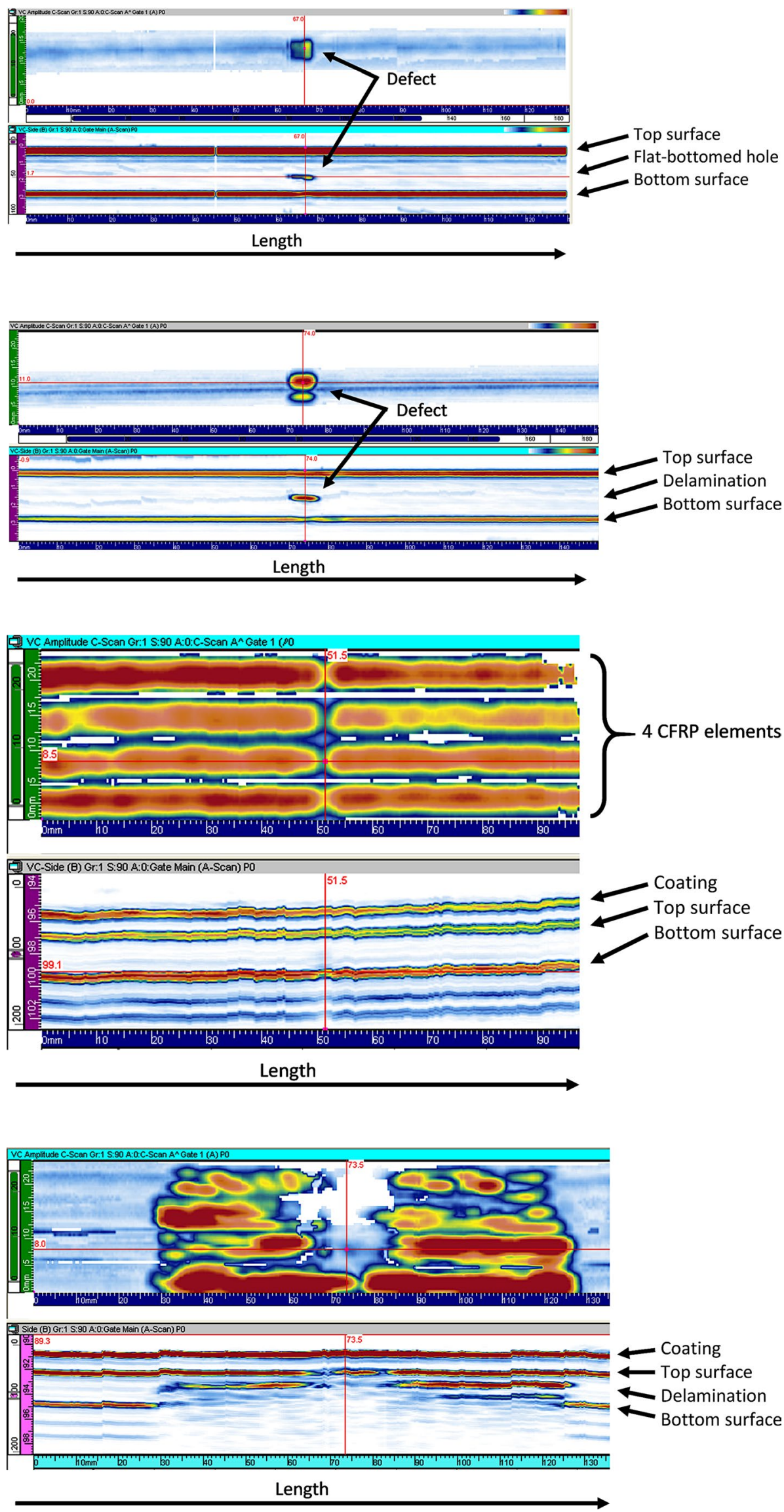
isotropic conditions are assumed (aluminium). The current field is condensed near the excitation coil and is symmetric relative to it. There is a noticeable edge effect in the transversal excitation. Edge effect is a phenomenon that occurs when an inspection coil is at the end of the test piece. In these instances, eddy current flow is distorted, as currents cannot flow at the edge [24].

The carbon fiber material properties used for the simulation were the measured electrical conductivity (Table 1) and a relative magnetic permeability of $\mu_{r}=1$.

Figure 13 shows the field of eddy current density in CFRP with different excitation orientations. Four loops are created in the edges of the element and away from the excitation when a transversal excitation is used (Fig. 13a). By applying a small angle $\left(5^{\circ}\right)$ to the excitation, it is possible to observe a preferred flow direction, which is the most conductive direction ( $\mathrm{Z}$ direction) of the CFRP element. Increasing this angle will facilitate this current flow as depicted. With CFRP, the excitation spreads along the element and its direction is not as distinct as in an isotropic material.

\subsection{Probe Design}

Two probe configurations were designed, produced and validated experimentally for ECT. Probe \#1 comprises of one excitation coil composed of $0.1 \mathrm{~mm}$ copper wire with 30 windings and two sensing helicoidal coils composed of $0.224 \mathrm{~mm}$ copper wire with 20 windings. The excitation windings are transversal to the CFRP elements with a length of twice the width of the element. On each side of the excitation winding there is one helicoidal coil which covers $90 \%$ of the element width as shown in Fig. 14. This probe will operate in reflection mode with a differential reading between the two sensing coils.

Probe \#2 comprises of four excitation coils composed of $0.1 \mathrm{~mm}$ copper wire with 30 windings and two sensing
Fig. 11 Geometrical model used in the simulation of the eddy current induced by the excitation filament of the ECT probe: a geometric model; b mesh representation
Fig. 12 Field of eddy current density on an isotropic material (Al) with different excitation orientations: a Excitation with $90^{\circ}$; b excitation with $45^{\circ}$; c excitation with $0^{\circ}$
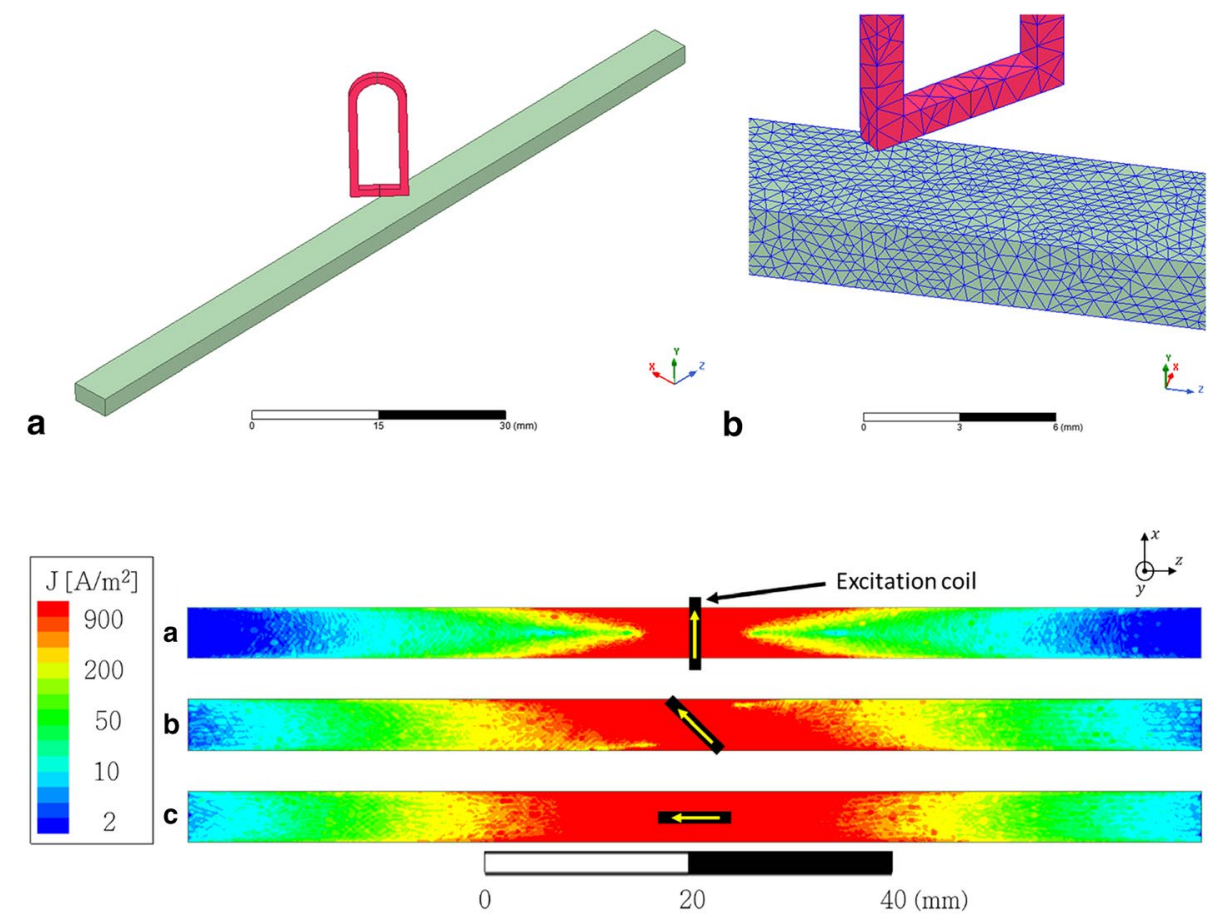

Fig. 13 Field of eddy current density in an anisotropic CFRP material with different excitation orientations: a excitation with $90^{\circ}$; b excitation with $85^{\circ}$; c Excitation with $45^{\circ}$; d Excitation with $0^{\circ}$

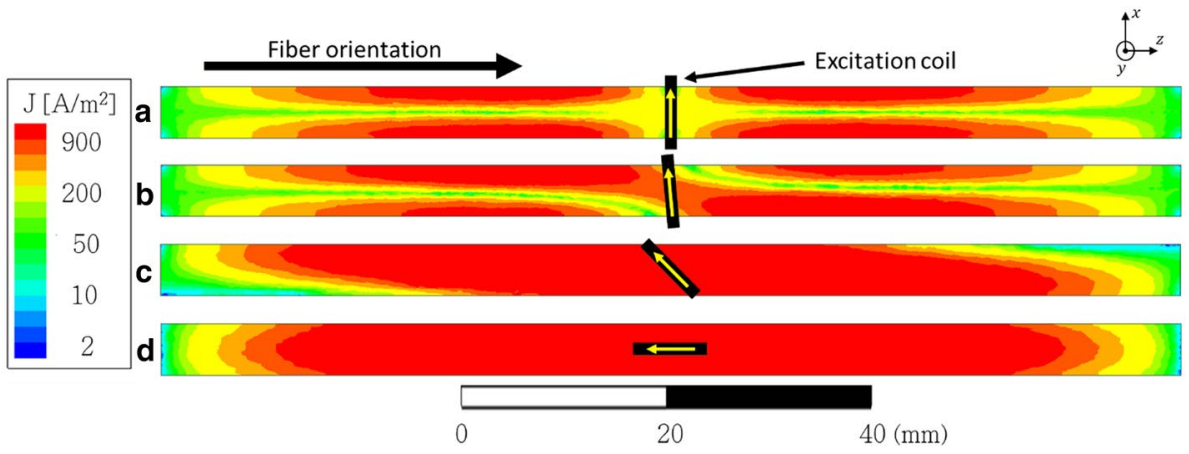



probe \#1: a isometric view of the probe design positioned on the right-side carbon fiber probe design; $\mathbf{c}$ top view of the probe design; $\mathbf{d}$ picture of the produced probe
Fig. 14 Description of the ECT element; $\mathbf{b}$ side view of the
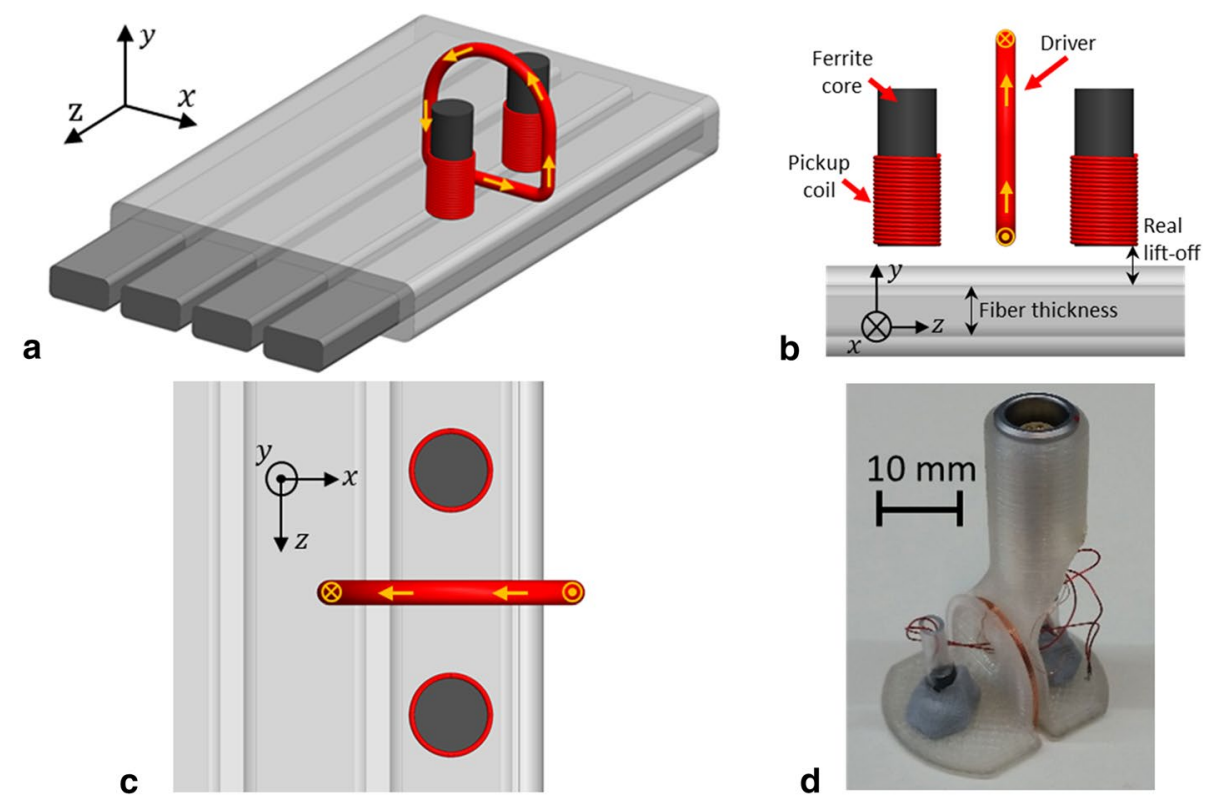

Fig. 15 Description of the ECT probe \#2: a isometric view of the probe design positioned on the right-side carbon fiber element; $\mathbf{b}$ side view of the probe design; $\mathbf{c}$ top view of the probe design; $\mathbf{d}$ picture of the produced probe

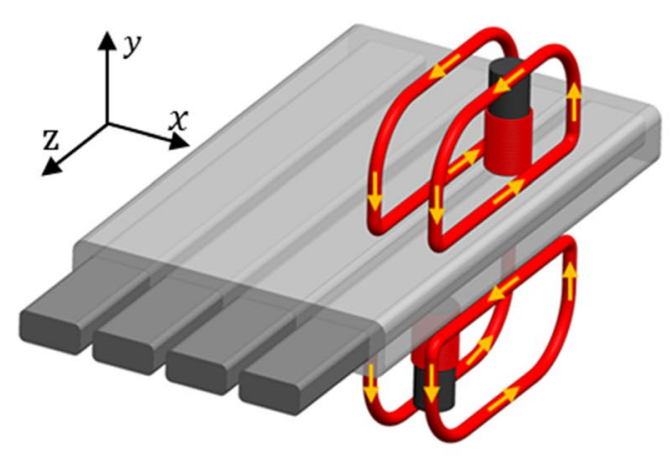

a
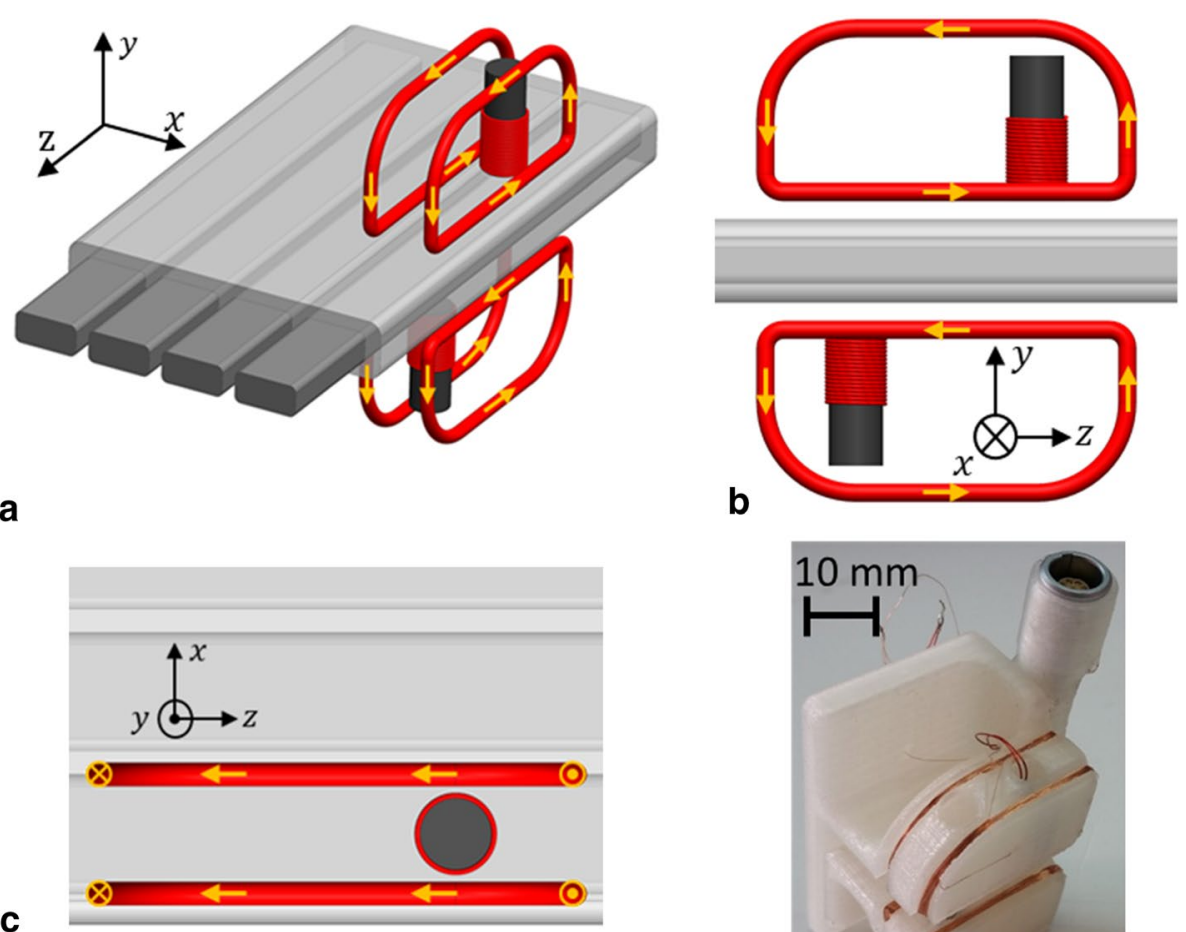

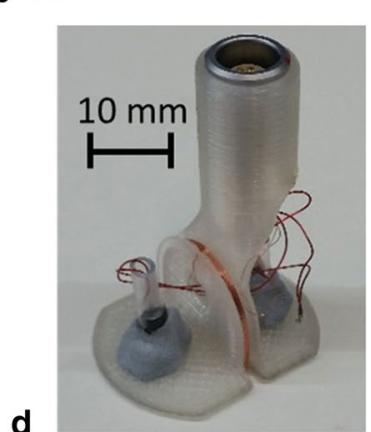

d

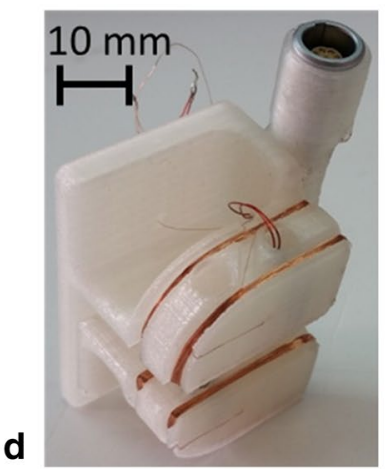

helicoidal coils composed of $0.224 \mathrm{~mm}$ copper wire with 20 windings (Fig. 15). There are two excitation windings on the top of the element along each edge, and two on the bottom (one along each edge) and all four windings are parallel to the CFRP rods. All four windings have the same winding direction that will create eddy current in the material in the same direction at the top and opposite direction at the bottom. This will force a closed eddy current loop inside the element. The probe also has two sensing coils but one at the top and one at the bottom, which will operate in a differential mode. This probe will operate in reflection mode with a differential reading between the two sensing coils. 


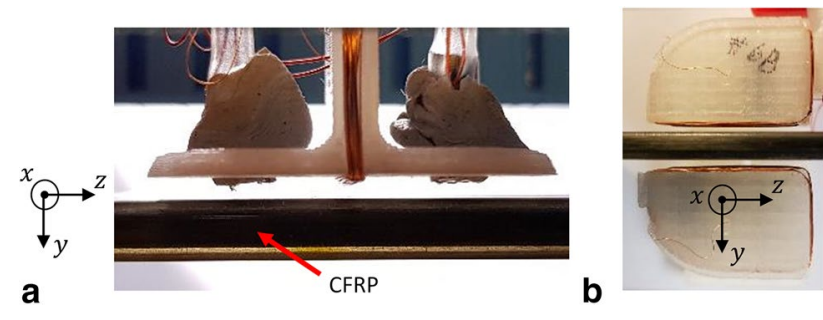

Fig. 16 ECT probes lift-off: a ECT probe \#1; b ECT probe \#2

\subsection{ECT Experimental Implementation}

The experimental implementation of the custom-made ECT probes was performed by means of an automated scanning device [25] responsible for the carbon fiber rope system movement while the probe remains stationary. Each one of the four CFRP elements was inspected one at a time. The movement, as well as the signal acquisition, were controlled and programmed in LabVIEW environment. Between each acquisition point, the sample moved $\Delta \mathrm{Z}=500 \mu \mathrm{m}$. The equipment responsible for the excitation and signal processing was an Olympus Nortec 500. The sampling rate of the equipment is $6 \mathrm{kHz}$. Absolute commercial pencil probes from various manufacturers were tested under the same inspection conditions without success. The distance between the probe and the carbon fiber rope system was about $3 \mathrm{~mm}$.

Figure 16 shows the lift-off conditions for both probes, i.e. probe distance to the inspected component.

Figure 17 depicts one scanned CFRP element with the sawed defect (Defect C) using EC probe \#1 at $4 \mathrm{MHz}$ in reflection mode and a commercial pencil probe at $6 \mathrm{MHz}$ in absolute mode. The output signal of probe \#1 follows the well-known shape due to the differential sensing arrangement. The sample with the $15 \mathrm{~J}$ impact damage was inspected with the same probes and operating parameters. This sample was longer, which allows identifying how high the signal-to-noise ratio is (Fig. 18).

Although ECT probe \#1 presented very good results, defects were not detected when assessed from the other side of the CFRP element. Thus, there was a need to develop a two-sided probe (EC probe \#2). The same two samples were inspected with $\mathrm{EC}$ probe \#2. This probe also operates in
Fig. 17 Output signal of ECT probe \#1 inspecting the saw cut sample (defect C) at $4 \mathrm{MHz}$, assessed from the defective side
Fig. 18 Output signal of ECT probe \#1 inspecting the impact damage sample (defect D) at $4 \mathrm{MHz}$, assessed from the defective side

Fig. 19 Output signal of ECT probe \#2 inspecting the saw cut sample (defect C) at $4 \mathrm{MHz}$
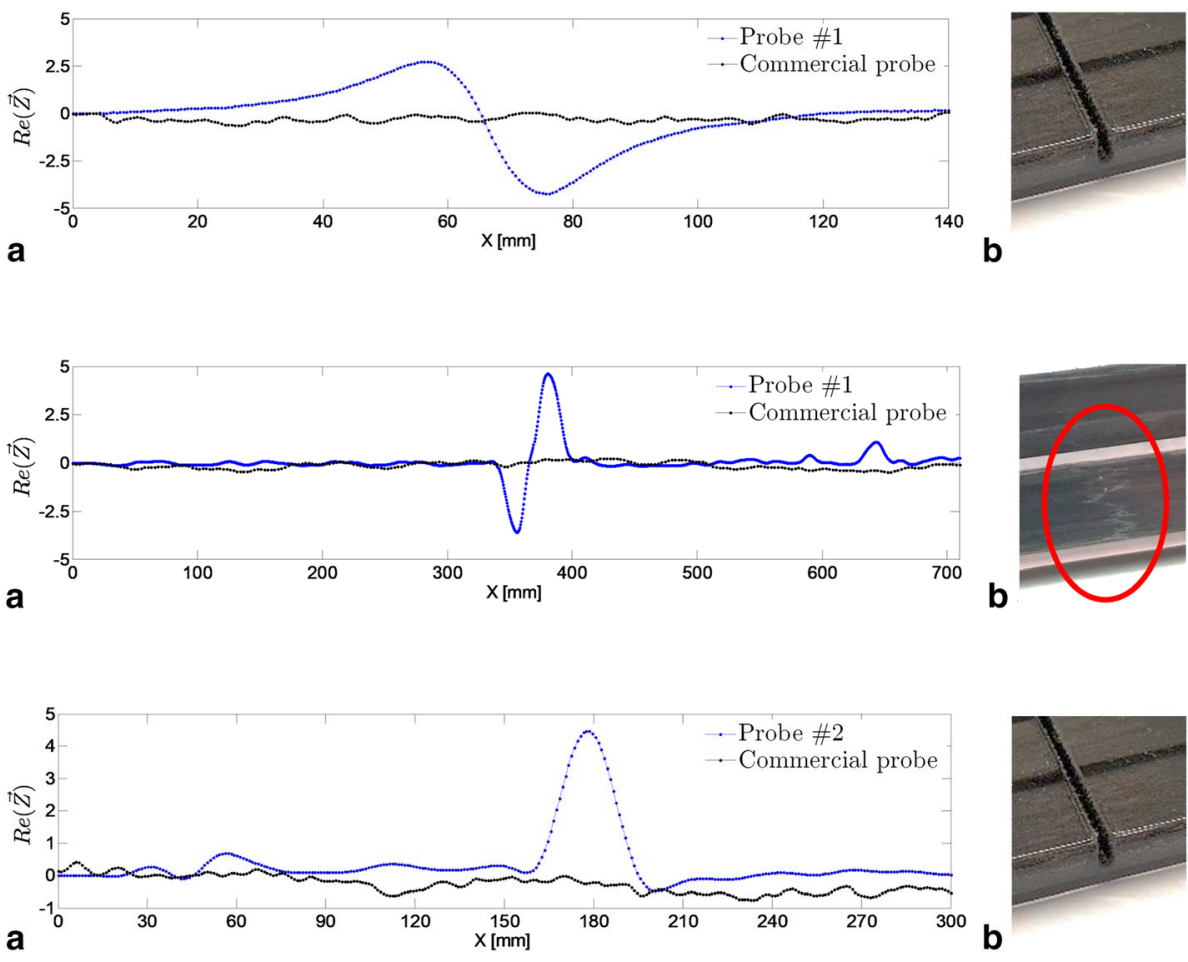

Fig. 20 Output signal of ECT probe \#2 inspecting the impact damage sample (defect D) at $4 \mathrm{MHz}$
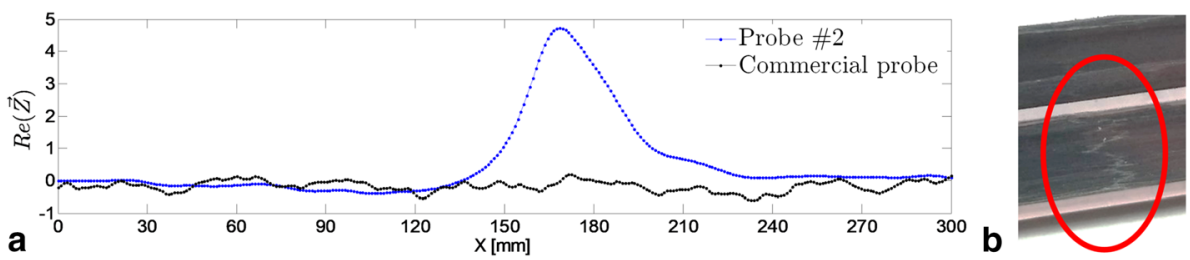
reflection mode and the test were done at 4 MHz. In Figs. 19 and 20 are presented the results for the sawed defect and impact defect respectively.

\section{Conclusions}

The requirements for online monitoring of carbon fiber composite ropes are challenging because several types of damage need to be detected and localised with fast and contactless inspection. In addition, information on damage size and morphology would help estimating the remaining service life, e.g., via modelling.

Thermography via TSA is able to detect the size and location of both fiber damage and delaminations. However, accuracy of the method is based on a large number of loading cycles of the same field-of-view, making it slow for online monitoring purposes. In addition, TSA works only for bare CFRP rods whereas the protective polymer coating, covering the rope, blocks infrared radiation and thus prevents monitoring rapid changes in surface temperature of the structural CFRP element.

Ultrasonic testing using phased-array probes can be used for inspection of coated ropes and it gives information on damage size and location. However, the coating causes significant attenuation making it crucial to obtain good coupling between the probe and rope surface. In practice, water coupling is needed which makes ultrasonic testing cumbersome to apply in field conditions.

Eddy current testing (ECT) can, however, detect various types of fiber damage at high inspection speeds, high signalto-noise ratios and provides some indication of the extent of damage via signal amplitude. It requires no contact and is insensitive to non-conductive coatings. ECT is more sensitive to defects located near the surface, since current density is largest at the surfaces.

As commercially available ECT probes were insufficient for inspecting the highly anisotropic CFRP material, two new dedicated probes were developed based on results from numerical simulations. The probe technology is relatively inexpensive and opens up opportunities for developing application-specific probes. Future research will focus on optimising these ECT probes based on the physical properties of unidirectional CFRP. The high data acquisition rate and inexpensive probe construction allows high-speed inspection of multiple ropes simultaneously during operation. It also produces an abundance of simple impedance data, which can be automatically filtered and analysed without the need of human interpretation. Eddy current testing is therefore a promising technique for real-time online monitoring of carbon fiber ropes while other techniques can provide complementary information once the damage location is known.
Acknowledgements Open access funding provided by Aalto University. KNA would like to acknowledge the Finnish Funding Agency for Technology and Innovation (TEKES). MAM acknowledge Fundação para a Ciência e a Tecnologia (FCT-MCTES) for its financial support via the PhD scholarship FCT-SFRH/BD/108168/2015. TGS acknowledge FCT-MCTES for the financial support in the scope of PEst-OE/ EME/UI0667/2014 (UNIDEMI).

Open Access This article is distributed under the terms of the Creative Commons Attribution 4.0 International License (http://creativeco mmons.org/licenses/by/4.0/), which permits unrestricted use, distribution, and reproduction in any medium, provided you give appropriate credit to the original author(s) and the source, provide a link to the Creative Commons license, and indicate if changes were made.

\section{References}

1. Brönniman, R., Nellen, P.M., Sennhauser, U.: Application and reliability of a fiber optical surveillance system for a stay cable bridge. Smart Mater. Struct. 7, 229-236 (1998)

2. Rebel, G., Verreet, R., Ridge, I.M.L.: Lightweight ropes for lifting applications. In: Proceedings of OIPEEC Conference, pp. 33-54. Athens, March (2006)

3. Mironenko, A., Sukhorukov, V.: Non-destructive testing of steel wire ropes in Russia. Insight 40, 395-397 (1998)

4. Schmieder, A., Heinze, T., Michael, M.: Failure analysis of high-strength fiber ropes. Mater. Sci. Forum 825-826, 891-898 (2015). https://doi.org/10.4028/www.scientific.net/ msf.825-826.891

5. Wang, X., Chung, D.D.L.: Self-monitoring of fatigue damage and dynamic strain in carbon fiber polymer-matrix composite. Composites B 29, 63-73 (1998)

6. Antin, K.-N., Harhanen, L., Bossuyt, S.: Damage detection in CFRP components using DIC. Adv. Opt. Methods Exp. Mech. 3, 57-62 (2016)

7. Schumacher, D., Antin, K.-N., Zscherpel, U., Vilaca, P.: Application of different X-ray techniques to improve in-service carbon fiber reinforced rope inspection. J Nondestruct Eval 36(4), $62(2017)$

8. Irving, P.E., Thiagarajan, C.: Fatigue damage characterization in carbon fibre composite materials using an electrical potential technique. Smart Mater. Struct. 7, 456-466 (1998). https://doi. org/10.1088/0964-1726/7/4/004

9. Rebel, G., Chaplin, C.R., Groves-Kirkby, C., Ridge, I.M.L.: Condition monitoring techniques for fibre mooring ropes. Insight 42(6), 36 (2000)

10. Benmokrane, B., Ali, A.H., Mohamed, H.M.: Durability performance and service life of CFCC tendons exposed to elevated temperature and alkaline environment. J. Compos. Constr. 20, 1-13 (2015). https://doi.org/10.1061/(asce)cc.1943-5614.0000606

11. Spinelli, G., Lamberti, P., Tucci, V., Vertuccio, L., Guadagno, L.: Experimental and theoretical study on piezorestistive properties of a structural resin reinforced with carbon nanotubes for strain sensing and damage monitoring. Composites B 145, 90-99 (2018). https://doi.org/10.1016/j.compositesb.2018.03.025

12. Meola, C., Boccardi, S., Carlomagno, G.M., Boffa, N.D., Monaco, E., Ricci, F.: Nondestructive evaluation of carbon fiber reinforced composites with infrared thermography and ultrasonics. Compos. Struct. 134, 845-853 (2015). https://doi. org/10.1016/j.compstruct.2015.08.119

13. Emery, T.R., Dulieu-Barton, J.M.: Thermoelastic stress analysis of damage mechanisms in composite materials. Composites 
A 41, 1729-1742 (2009). https://doi.org/10.1016/j.composites a.2009.08.015

14. Freire, J.L.F., Waugh, R.C., Fruehmann, R., Dulieu-Barton, J.M.: Using thermoelastic stress analysis to detect damaged and hot spot areas in structural compoents. J. Mech. Eng. Autom. 5, 623-634 (2015). https://doi.org/10.17265/2159-5275/2015.11.005

15. Almeida, G., Gonzalez, J., Rosado, L., Vilaça, P., Santos, T.G.: Advances in NDT and materials characterization by eddy currents. In: Proceedings of 46th CIRP Conference on Manufacturing Systems, Portugal, May, pp. 359-364 (2013). https://doi. org/10.1016/j.procir.2013.05.061

16. Heuer, H., et al.: Review on quality assurance along the CFRP value chain-non-destructive testing of fabrics, preforms and CFRP by HF radio wave techniques. Composites B 77, 494-501 (2015). https://doi.org/10.1016/j.compositesb.2015.03.022

17. Mook, G., Lange, R., Koeser, O.: Non-destructive characterisation of carbon-fibre-reinforced plastics by means of eddycurrents. Compos. Sci. Technol. 61, 865-873 (2001)

18. Cheng, J., et al.: Application of low frequency ECT method in noncontact detection and visualization of CFRP material. Composites B 110, 141-152 (2017). https://doi.org/10.1016/j.compo sitesb.2016.11.018

19. Schmidt, C., Schultz, C., Weber, P., Denkena, B.: Evaluation of eddy current testing for quality assurance and process monitoring of automated fiber placement. Composites B 56, 109-116 (2014). https://doi.org/10.1016/j.compositesb.2013.08.061

20. Cheng, J., Ji, H., Qiu, J., Takagi, T., Uchimoto, T., Hu, N.: Role of interlaminar interface on bulk conductivity and electrical anisotropy of CFRP laminates measured by eddy current method. NDT E Int. 68, 1-12 (2014). https://doi.org/10.1016/j.ndtei nt.2014.07.001

21. Koyama, K., Hoshikawa, H., Hirano, T.: Investigation of impact damage of carbon fiber-reinforced plastic (CFRP) by eddy current non-destructive testing. In: Conference on Smart Materials, Structures \& NDT in Aerospace. Quebec, November (2011)

22. ISO 14125:1997. Fibre-reinforced plastic composites-determination of apparent interlaminar shear strength by short-beam method

23. ASTM D5628-10. Standard test method for impact resistance of flat, rigid plastic specimen by means of a falling dart (Tup or falling mass)

24. Martín, J.: Non-destructive techniques based on eddy current testing. Sensors 11, 2525-2565 (2011). https://doi.org/10.3390/s1103 02525

25. Machado, M.A., Antin, K.-N., Rosado, L., Vilaca, P., Santos, T.G.: Contactless high-speed eddy current inspection of unidirectional carbon fiber reinforced polymer. Compos. B Eng. 2019(168), 226235 (2019). https://doi.org/10.1016/j.compositesb.2018.12.021 